\title{
Thymic Carcinoma Associated with Cerebellar Degeneration
}

\author{
Irene García Cuartero ${ }^{1}$, Monica Rodríguez Galdeano ${ }^{2}$, Montserrat Pérez Pinar², Julián Solís García del Pozo $^{2}$ \\ ${ }^{1}$ Department of Internal Medicine,A Ibacete University Hospital, Albacete, Spain \\ ${ }^{2}$ Department of Internal Medicine, Villarrobledo General Hospital, Villarrobledo, Albacete, Spain
}

Doi: 10.12890/2018_000780-European Journal of Case Reports in Internal Medicine - @ C EFIM 2018

Received: $11 / 11 / 2017$

Accepted: 01/12/2017

Published: 05/01/2018

How to cite this article: García Cuartero I, Rodríguez Galdeano M, Pérez Pinar M, Solís García del Pozo J. Thymic carcinoma with cerebellar degeneration. EJCRIM 2018;5: doi:10.12890/2018_000780

Conflicts of Interests: The Authors declare that there are no competing interests.

This article is licensed under a Commons Attribution Non-Commercial 4.0 License

\section{ABSTRACT}

We present the case of a 57-year-old man with ataxia and clinical and radiological features of cerebellar degeneration. Computed tomography showed a mediastinal mass and the patient was diagnosed with thymic carcinoma. Paraneoplastic cerebellar degeneration is aninfrequent disorder and its association with thymic carcinoma very rare.

\section{LEARNING POINTS}

- Unexplained subacute neurological symptoms in an adult patient should beconsidered in the possibility of a paraneoplastic syndrome. In patients over 50 years of age, acute or subacute cerebellar degeneration is paraneoplastic in $50 \%$ of cases.

- Small-cell lung cancer is the most common cancer-causing paraneoplastic cerebellar degeneration (PCD). Despite this, mediastinal tumours such as thymus neoplasms should not be ruled out in the differential diagnosis.

- Antineuronal antibodies are not detected in $40 \%$ of patients with PCD, sothe exclusion of other aetiologies or the demonstration of cancer formsthe basis of the final diagnosis.

\section{KEYWORDS}

Thymic carcinoma; cerebellar degeneration, paraneoplastic syndrome

\section{CASE DESCRIPTION}

A 57-year-old man was referred to our service for ataxic gait. For over 1 year he had been experiencing a progressive gait disorder, characterised by instability without clear lateropulsion and no objective vertigo. In the previous few months, he had also reported associated dysarthria.

Neurological examination showed dysarthric speech, mild left finger-to-nose dysmetria, left dysdiadochokinesia and bilateral heel-knee dysmetria. Broad-based ataxic gait, inability to tandem walk, and balance changes were also observed,as the patient could not stand on one leg. Brain MRI showed marked cerebellar atrophy together with brainstem atrophy.

Standard biochemical tests, blood count and coagulation profile were not remarkable. Folic acid and vitamin B12 were normal. The values of vitamin E and biotin were low. Serology studies for syphilis, Brucella sp., Borrelia sp., HBV, HCV and HIV were negative. Tumour markers were negative, apart from CEA of $11.4 \mathrm{ng} / \mathrm{mL}$. Onconeural antibodies (anti-amphiphysin, anti-CV2/CRMP5, anti-PNMA2/Ma2/Ta, anti-Ri/ ANNA-2, anti-Yo/PCA1 and anti-Hu/ANNA-1) were negative. A chest CT scan revealed a mass in the anterior mediastinal space (Fig. 1). A pulmonary intraparenchymal nodule of $0.5 \mathrm{~cm}$ in the left lower lobe was also seen along with mediastinal adenopathies. 
A mediastinoscopy demonstrated a solid anterior mediastinal mass and a node in the internal mammary lymph node chain. The histological study findings of the mediastinal mass reported poorly differentiated carcinoma with areas of small-cell carcinoma, characterised by remnants of thymic tissue with the presence of artefacts. Immunohistochemistry was positive for CD5 and CD117. Therefore, a thymic carcinoma was diagnosed (Fig. 2).

Although the vitamin E and biotin levels returned to normal, the clinical neurological signs and symptoms did not improve. In spite of the negative results of the onconeural antibodies, the most likely diagnosis was cerebellar degeneration in the context of thymic carcinoma.

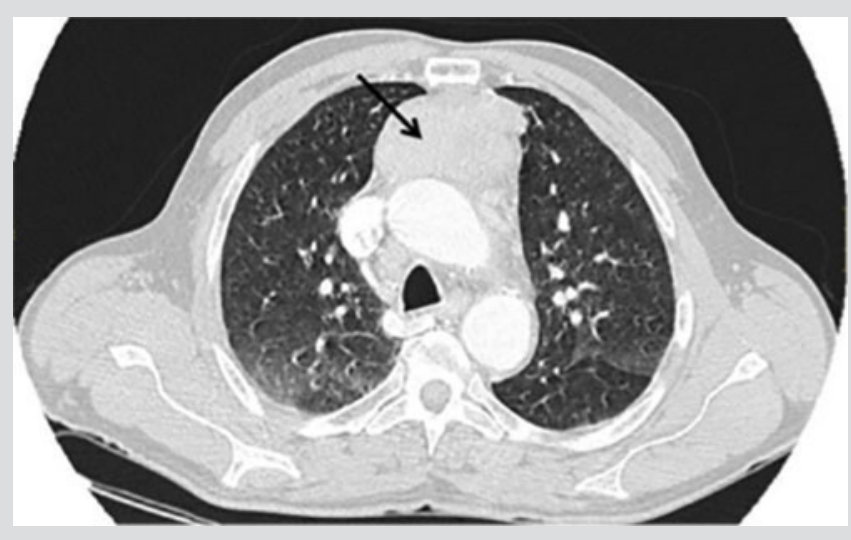

Figure 1. Computed tomography revealing a mass in the anterior mediastinal space

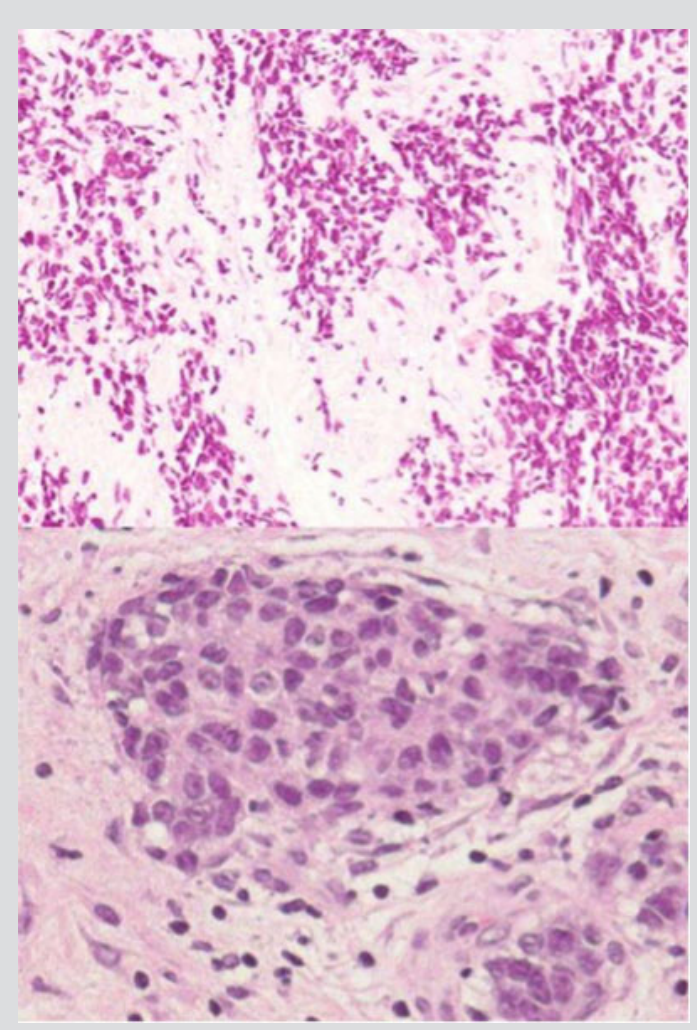

Figure 2.Groups of epithelial cells with extensive cytoplasm and nuclei showing nucleoli; $H \& E, \times 40$ (top). Component of small-cell undifferentiated carcinoma showing nuclear artefacts; H\&E, $\times 20$ (bottom)

\section{DISCUSSION}

Thymomas or thymic carcinomas occurinfrequentlyinneoplasms of the anterior mediastinum, accounting for less than $1.5 \%$ of all tumours.Of these, thymic carcinomas are extremely rare, comprising $0.06 \%$ of thymic neoplasms ${ }^{[1]}$. The differential diagnosis includes neuroendocrine tumours, germ cell tumours, lymphomas, stromal tumours, thymic hyperplasia, thymic cysts, and lung cancer. The usual age of onset is between 50 and 60 years ${ }^{[2]}$. Approximately $30 \%$ of patients are asymptomatic at diagnosis and the tumour is detected as an occasional finding in a chest X-ray study performed for another reason. In the remainder of cases, onset occurs primarily with cough, chest pain or dyspnoea. The diagnosis of thymic carcinoma does not usually require histochemical analysis, although this may be useful in differentiating primary thymic carcinomas from metastatic carcinomas from other localisations, predominantly the lung. This distinction may be facilitated by the use of immunohistochemical markers CD5 and CD117, which, although not completely specific, are typically expressed in a high percentage of thymic carcinomas ${ }^{[2]}$.

Paraneoplastic neurological syndromes are a heterogeneous group of disorders occurring in patients with malignancy ${ }^{[3]}$. Also, paraneoplastic syndromes may predate the presentation with thymoma, be diagnosed concurrently with the thymic tumour, or occur after treatment (with or without evidence of tumour recurrence). They are not related to the metastatic invasion of cancer or other complications such as infections, coagulation disorders, nutritional or metabolic deficiencies or to the effects of radiotherapy or chemotherapy, but rather are 
immune-mediated. They usually cause greater disability than the neoplastic process itself. The most common paraneoplastic syndrome associated with thymoma is myasthenia gravis. However, a wide range of other autoimmune paraneoplastic syndromes has been reported. Among them are neuromuscular disorders (such as myotonic dystrophy, limbic encephalitis or peripheral neuropathy),haematological disorders (such as red cell aplasia, pernicious anaemia, erythrocytosis, pancytopenia or haemolytic anaemia), collagen and autoimmune disorders, dermatological disorders (such as pemphigus or lichen planus), hypogammaglobulinaemia and other immune deficiency disorders, endocrine disorders (such as multiple endocrine neoplasia, Cushing syndrome orthyroiditis) and other disorders such as giant cell myocarditis, nephrotic syndrome, ulcerative colitis or hypertrophic osteoarthropathy ${ }^{[2]}$.

Paraneoplastic cerebellar degeneration (PCD) is a rare disorder characterised clinically by subacute and progressive cerebellar dysfunction with signs and symptoms of vertigo, vomiting, dysarthria, diplopia and ataxia. These often precede the diagnosis of a potentially treatable tumour by months or years ${ }^{[4]}$. Small-cell lung cancer is the most common cancer-causing PCD. Several antibodies have been identified in patients with PCD with different prognostic implications and treatment responses:anti-Yo, anti-Ri, anti-Tr, anti-Hu, anti-glutamate receptor ${ }^{[4]}$. Almost $40 \%$ of patients with PCD do not show antineuronal antibodies, and the final diagnosis is based on the exclusion of other aetiologies or the demonstration of cancer $^{[3]}$.

In our case, the patient experienced progression of his clinical neurological signs and symptoms for a prolonged period before diagnosis. Although the vitamin $\mathrm{E}$ and biotin deficiencies present in our patient may be the cause of acquired cerebellar ataxia, the fact that the clinical signs and symptoms did not improve when their levels returned to normal rules out this aetiology. In spite of the negative results of the onconeural antibody tests, the coincidence of PCD and thymic carcinoma in this patient suggestsarelationship between them. To our knowledge,this coincidence has only rarely been reported. We have found only a few isolated clinical cases with this association published ${ }^{[5-7]}$.

\section{REFERENCES}

1. Greene MA, Malias MA. Aggressive multimodality treatment of invasive thymic carcinoma. J Thorac Cardiovasc Surg 2003;125:434-36.

2. Girard N, Ruffini E, Marx A, Faivre-Finn C,Peters S, on behalf of the ESMO GuidelinesCommittee. Thymic epithelial tumours: ESMO Clinical Practice Guidelines for diagnosis, treatment and follow-up. Ann Oncol 2015;26(Suppl 5):v40-v55.

3. Höftberger R, Rosenfeld MR, Dalmau J. Update on neurological paraneoplastic syndromes. Curr Opin Oncol 2015;27:489-95.

4. Klein JP, Stover DG, Oxnard GR, Levy BD, Loscalzo J. Restoring balance: paraneoplastic cerebellar degeneration. Am J Med 2017;130:e85-e87.

5. Yamaguchi Y, Wada M, Tanji H, Kurokawa K, Kawanami T, Ohtake H, et al. Marked improvement in opsoclonus and cerebellar ataxia after the surgical removal of a squamous cell carcinoma of the thymus: a case report. J Neurol Sci 2013;325:156-59.

6. Bataller L, Valero C, Díaz R, Froufe A, Garcia-Zarza A, Ribalta T, et al. Cerebellarataxia associated with neuroendocrine thymic carcinoma and GAD antibodies. J Neurol Neurosurg Psychiatry 2009;80:696-97.

7. Solà-Valls N, Gaba L, Muñoz E, Mellado B, Ribalta T, Saiz A, et al. Paraneoplastic cerebellar degeneration associated with thymic germinoma. J Neurol Sci 2012;320:153-55. 